\title{
PHENIX measurement of direct photon-triggered two-particle correlations in heavy ion collisions and its implication for medium-induced energy loss.
}

\author{
Alexandre Lebedev* ${ }^{\dagger}$ \\ Iowa State University \\ E-mail: lebedeveiastate.edu
}

\begin{abstract}
Direct photon-hadron correlations are an excellent probe for QCD effects, including parton energy loss in the Quark-Gluon Plasma. At leading order, direct photons balance the $p_{T}$ of the away-side jet. In addition, as a colorless probe, direct photons do not interact strongly with the colored medium providing a less biased trigger than a single high- $p_{T}$ hadron. PHENIX has measured direct photon-triggered two-particle azimuthal correlations in a variety of collision systems at $\sqrt{s}=200 \mathrm{GeV}$. In $\mathrm{d}+\mathrm{Au}$ collisions, no modification of the per-trigger pair yields compared to $\mathrm{p}+\mathrm{p}$ collisions was observed constraining the amount of cold nuclear matter effects in such collisions. In A+A collisions, direct photons have been identified statistically, as well as using an isolation cut. Combining data sets from different collisions systems allows us to quantify the transition from suppression at high $z_{T}\left(=p_{T}^{h} / p_{T}^{\gamma}\right)$ to the enhancement of low $z_{T}$ particles relative to $\mathrm{p}+\mathrm{p}$, and to study this transition as a function of trigger $p_{T}$. Integrating per-trigger yields in different ranges of the away-side gives insights on the redistribution of energy within the jet. The implication for our understanding of energy loss mechanisms will be discussed.
\end{abstract}

International Conference on Hard and Electromagnetic Probes of High-Energy Nuclear Collisions 30 September - 5 October 2018

Aix-Les-Bains, Savoie, France

\footnotetext{
${ }^{*}$ Speaker.

${ }^{\dagger}$ For the PHENIX Collaboration.
} 


\section{Introduction}

Experiments and theoretical analyses have shown that a plasma of quarks and gluons created in relativistic heavy ion collisions exhibits remarkable properties, including opacity to traversing quarks and gluons. However, the exact mechanism for energy loss by these partons in quark gluon plasma and transport of the deposited energy within the plasma is not yet well understood. Experimental probes to address this question include correlations among particles arising from initial hard partonic scattering. Correlations of direct photons with hadrons are of particular interest.

Direct photons do not interact via the strong force and are produced via the quantum chromodynamics analog of Compton scattering at leading order. In the limit of negligible initial transverse momentum, the final state quark and photon are emitted back-to-back in azimuth with the photon balancing the transverse momentum of the jet arising from the quark. Consequently, measuring the correlation of high momentum direct photons with opposing hadrons allows investigation of quark gluon plasma effects upon transiting quarks and their fragmentation into jets of hadrons.

In this talk we present new PHENIX results on direct photon-hadron correlations in d+Au and $\mathrm{Au}+\mathrm{Au}$ collisions at $\sqrt{s}=200 \mathrm{GeV}$.

\section{Direct photon-hadron correlations measurement in PHENIX}

The measurements in this paper use the PHENIX central spectrometers [1]. Two particle correlations are constructed by pairing photons measured in the Electromagnetic Calorimeter (EMCal) with charged hadrons reconstructed in the Drift Chambers (DC) and Pad Chambers (PC) The acceptance in rapidity is $|\eta|<0.35$, while each spectrometer arm covers $90^{\circ}$ in azimuth. Beam-Beam counters $(\mathrm{BBC})$ located at 1.44 meters from the center of the interaction region, cover rapidity range from 3.0 to 3.9 and full azimuthal angle. They are used to determine the collision centralities and event vertex position.

We aim to quantify the modification of the jet fragmentation function $D(z)$ in $\mathrm{Au}+\mathrm{Au}$ and $\mathrm{d}+\mathrm{Au}$ collisions, compared to the $\mathrm{p}+\mathrm{p}$ baseline. The jet fragmentation function describes the probability of an outgoing parton to yield a hadron with momentum fraction $z=p_{\text {hadron }} / p_{\text {parton }}$. Assuming that the intrinsic $k_{T}$ of partons in a nucleon has a negligible effect, then $z_{T}=p_{T}^{h} / p_{T}^{\gamma}$ can be used to approximate $z$. In order to focus on the low $z_{T}$ region we use the variable $\xi=\ln \left(1 / z_{T}\right)$.

A more detailed description of the analysis procedure can be found in [2] and [3].

Fig. 1 shows azimuthal angular distributions of hadrons associated with direct photons of $5<p_{T}<7 \mathrm{GeV} / \mathrm{c}$, in the $0-10 \%$ most central Au+Au collisions (black circles) and in $\mathrm{p}+\mathrm{p}$ collisions (open blue squares). The plots are shown for different $\xi$ ranges.

The fragmentation function is obtained by integrating the per-trigger yield of hadrons in the azimuthal angle region $|\Delta \phi-\pi|<\pi / 2$ rad and is shown in Fig. 2 as a function of $\xi$ for $p+p, d+A u$ and $\mathrm{Au}+\mathrm{Au}$ collisions.

\section{The results}

$I_{A A}=Y_{A A} / Y_{p p} \approx D_{A A}(z) / D_{p p}(z)$, where $Y_{A A}$ and $Y_{p p}$ are per trigger yields of hadrons in nucleus-nucleus and proton-proton collisions correspondingly, is a nuclear modification factor 


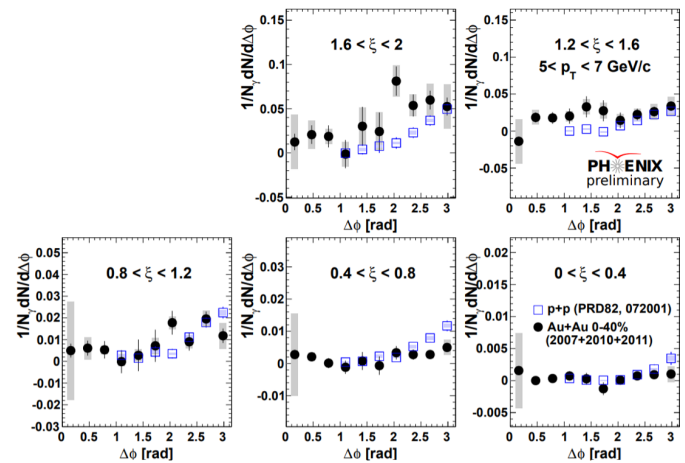

Figure 1: Per trigger yields of hadrons as a function of the angle between the hadron and the trigger photon.

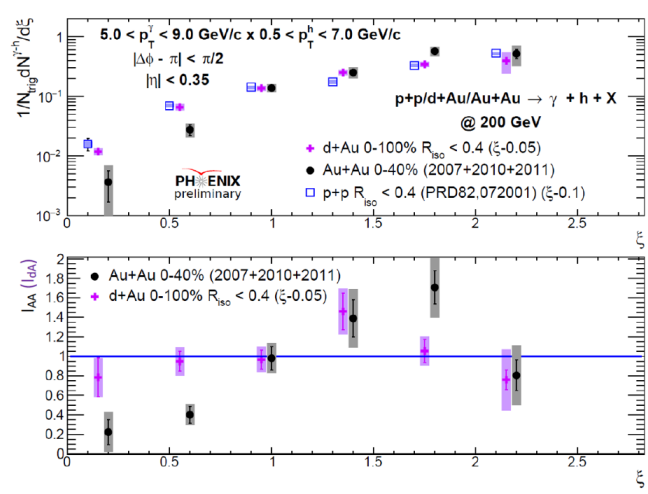

Figure 2: Effective fragmentation function (top) and nuclear modification factor (bottom) as a function of $\xi$.

which quantifies the difference between the fragmentation functions in $\mathrm{Au}+\mathrm{Au}$ and $\mathrm{p}+\mathrm{p}$. In the absence of in-medium modifications, $I_{A A}$ should be equal to 1 . The nuclear modification factor $I_{A A}$ is shown in Fig. 3 as a function of $\xi$ for three different trigger photon $p_{T}$ ranges. While the associated hadron yields are smaller than those in $\mathrm{p}+\mathrm{p}$ at low $\xi$, the appearance of extra particles at higher $\xi$ is observed for triggers with $p_{T}$ of $5-7 \mathrm{GeV} / \mathrm{c}$. A qualitatively similar increase of $I_{A A}$ with $\xi$ is visible for the $7-9 \mathrm{GeV} / \mathrm{c} p_{T}$ bin.

In order to investigate where the energy deposited in the plasma goes, we study the dependence of $I_{A A}$ on the integration range in azimuthal opening angle. The hadron yields are integrated in two narrower angular ranges on the away side $|\Delta \phi-\pi|<\pi / 3 \mathrm{rad}$ and $|\Delta \phi-\pi|<\pi / 6 \mathrm{rad}$. The resulting $I_{A A}$ values are shown in Fig. 4 for all three photon $p_{T}$ bins. The enhancement over $\mathrm{p}+\mathrm{p}$ is largest for 5-7 GeV/c triggers, and for the full away-side integration range. The suppression observed at low $\xi$ and low trigger photon $p_{T}$ is similar for the different integration regions, suggesting that the jet core is suppressed, and the enhancement exists at large angles.

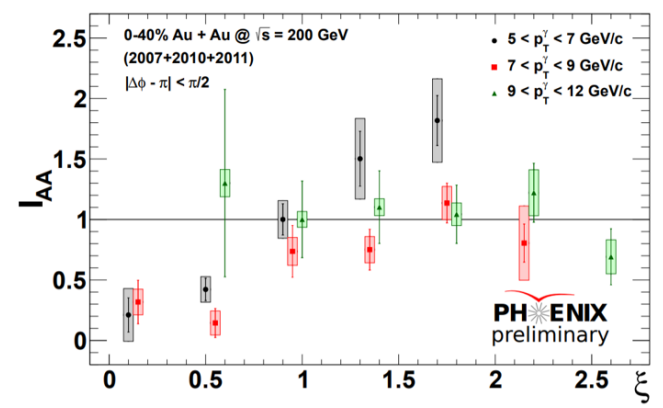

Figure 3: Two particle nuclear modification factor $I_{A A}$ as a function of $\xi$.
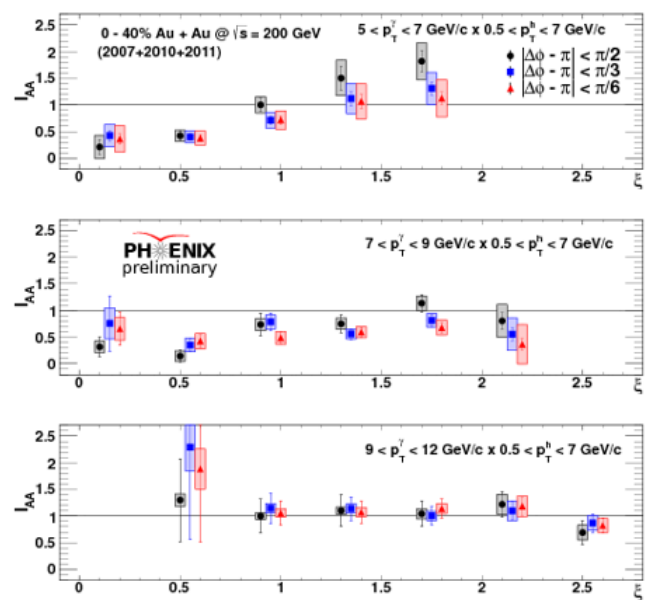

Figure 4: $I_{A A}$ as a function of $\xi$ for different trigger $p_{T}$ and integration range.

Whether or not $I_{A A}$ becomes significantly larger than unity - what we have been referring 
to as enhancement - there is a tendency for $I_{A A}$ to increase with increasing $\xi$. To quantify this, we calculate the weighted averages of $I_{A A}$ values above and below $\xi=1.2$. The ratio for each integration range is plotted in Fig. 5, as a function of the trigger photon $p_{T}$. The enhancement is largest for softer jets and for the full away-side integration range, implying that jets with lower energy are broadened more than higher energy jets.

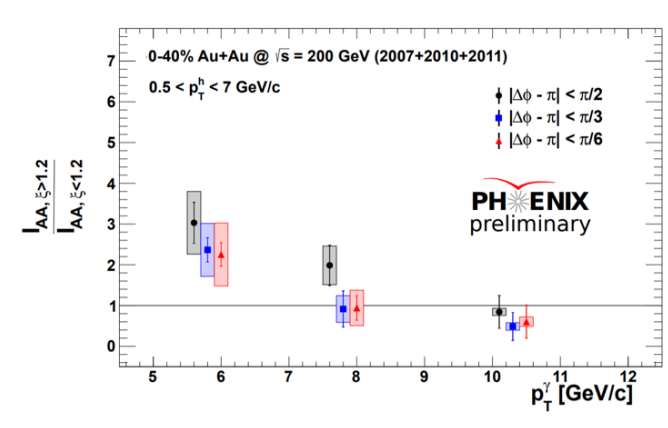

Figure 5: Ratio of $I_{A A}$ at large and small $\xi$ as a function of trigger $p_{T}$ for different integration ranges.

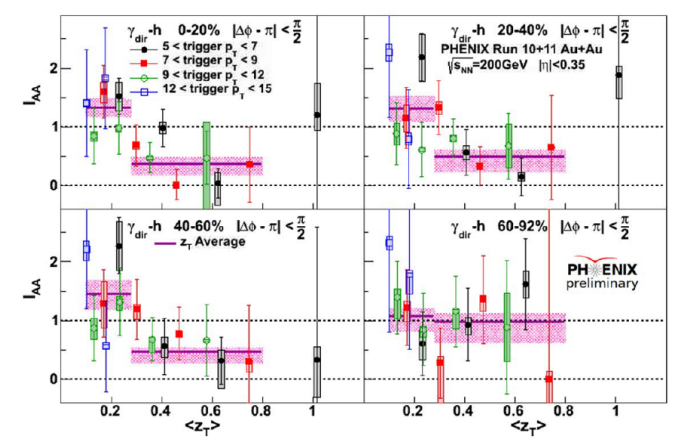

Figure 6: Average nuclear modification factor $I_{A A}$ as a function of $z_{T}$ for different centralities in $\mathrm{Au}+\mathrm{Au}$ collisions.

\subsection{Centrality dependence}

Adapting the isolation cone method for use in $\mathrm{Au}+\mathrm{Au}$ collisions allowed us to improve the precision of direct photon-hadron correlation measurements and determine the centrality dependence of the nuclear modification factor. Fig. 6 shows $I_{A A}$ as a function of mean $z_{T}$ for four different centrality bins. The nuclear modification factors $I_{A A}$ were averaged above and below $z_{T}=0.3$ as is shown by purple bands in this figure. These averages were used to study suppression/enhancement as a function of collision centrality.

The average $I_{A A}$ is shown in the top panel of Fig. 7 for two $z_{T}$ ranges as a function of centrality expressed as the number of participants $N_{\text {part }}$. While at low $z_{T}$ the average $I_{A A}$ is consistent with one, for the high $z_{T}$ bin there is a clear decrease of the average $I_{A A}$ with increasing centrality. The bottom panel of this figure shows the double ratio of $I_{A A}$ at low and high $z_{T}$. A statistically significant monotonic increase in suppression is seen as the collision centrality increases.

Fig. 8 shows the comparison of the average two-particle nuclear modification factor $I_{A A}$ and the single particle nuclear modification factor $R_{A A}$ for $\pi^{0}$ in Au+Au collisions as a function of centrality. Similar suppression is observed in both cases.

\section{Conclusions}

Direct photon-hadron correlations are a powerful tool for studying QCD.

$\mathrm{d}+\mathrm{Au}$ collisions show no significant modification of fragmentation function compared to $\mathrm{p}+\mathrm{p}$, which indicates that possible CNM effects are small.

In $\mathrm{Au}+\mathrm{Au}$ collisions enhancement at low $z_{T}$ (high $\xi$ ) and suppression at high $z_{T}$ (low $\xi$ ) is observed. Suppression increases monotonically with centrality. Enhancement is largest for a broad 


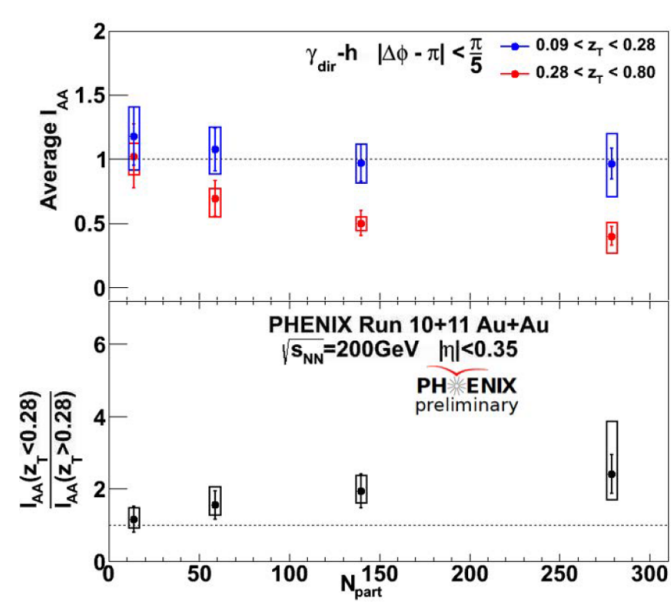

Figure 7: Average $I_{A A}$ as a function of centrality expressed as the number of participants for two different $z_{T}$ ranges (top) $I_{A A}$ double ratio (bottom).

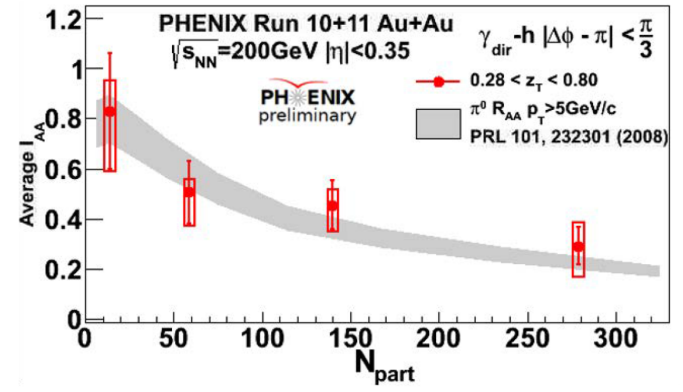

Figure 8: Comparison of average $I_{A A}$ for direct photon-hadron correlations and single particle nuclear modification factor $R_{A A}$ for $\pi^{0}$.

integration region and for soft hadrons. Transition from suppression to enhancement appears to occur at fixed hadron $p_{T}$. All this suggests medium response dominated processes.

Two-particle nuclear modification factor $I_{A A}$ for direct photon-hadron correations and single particle nuclear modification factor $R_{A A}$ for $\pi^{0}$ are consistent in $\mathrm{Au}+\mathrm{Au}$ collisions.

More measurements are expected to come from PHENIX soon. Large $\mathrm{Au}+\mathrm{Au}$ data sets from 2014 and 2016 are currently being analyzed.

\section{References}

[1] K. Adcox et al., NIM A499 469-479 (2003)

[2] A.Adare et al., Phys. Rev. C86 024909 (2012)

[3] A. Adare et al., Phys. Rev. C80 024908 (2009) 